\title{
Severe allergic asthma: Does alexithymia interfere with omalizumab treatment outcome?
}

\author{
Marta Liotta, ${ }^{1}$ Marco Liotta, ${ }^{2}$ Salvatore Saitta, ${ }^{3}$ Luisa Ricciardi ${ }^{4}$
}

\begin{abstract}
Background: Alexithymia is among psychological factors reported to interfere with asthma management. Severe allergic asthma (SAA) is characterized by uncontrolled asthma despite maximal standard pharmacological treatment which can benefit from an add-on treatment with Omalizumab, an anti-IgE monoclonal antibody.
\end{abstract}

Objectives: To evaluate if alexithymia influences the efficacy of omalizumab in SAA.

Methods: The total alexithymia score 20 (TAS 20) questionnaire allowed to detect alexithymia. SAA was monitored recording number of exacerbations, asthma control test (ACT) and asthma quality of life questionnaire (AQLQ) scores, as well as forced expiratory volumes in 1 second \% (FEV1\%) levels before starting omalizumab, 1 and 2 years after.

Results: The study was conducted on 18 patients; Group 1, TAS $20 \geq 61$, was of 2 males and 4 females with SAA and alexithymia, while Group 2 , TAS $20 \leq 51$, was of 8 males and 4 females, without alexithymia. Group 1 had a statistically significant decrease in asthma exacerbations " $p=0.004$ ", while ACT " $p=0.008$ " and AQLQ scores statistically increased. FEV1 values increased but not statistically significantly. Group 2 had a highly statistically significant decrease in the number of exacerbations and a highly statistically significant increase of ACT " $p<0.0001$ ", FEV1 “ $p=0.008 ”$ and AQLQ scores.

Conclusion: Regardless the presence or not of alexithymia, all patients with SAA obtained a marked improvement after starting treatment with omalizumab. Therefore alexithymia does not seem to influence the treatment outcome of omalizumab.

Key words: asthma, alexithymia, allergy, omalizumab, quality of life

\section{Citation:}

Liotta, M., Liotta, M., Saitta, S., Ricciardi, L. (2023)

Severe allergic asthma: Does alexithymia interfere with omalizumab treatment outcome? Asian Pac J Allergy Immunol, 41(1), 53-59. https://doi.org/10.12932/ap-040121-1031

\footnotetext{
Affiliations:

${ }^{1}$ Allergy and Clinical Immunology Unit, University of Messina, Messina, Italy

${ }^{2}$ Specialist in Psichology, Messina, Italy

${ }_{3}$ Messina Provincial Health Department, Messina, Italy

${ }^{4}$ Allergy and Clinical Immunology Unit, Department of Clinical and Experimental Medicine, University of Messina, Messina, Italy
}

\section{Corresponding author:}

Luisa Ricciardi

Allergy and Clinical Immunology Unit, Department of Clinical and Experimental Medicine, University of Messina,

G. Martino University School Hospital

Via Consolare Valeria 1, 98124 Messina, Italy

E-mail: lricciardi@unime.it

\section{Introduction}

Asthma is defined as a multi-factorial respiratory disease characterized by chronic airway inflammation and bronchial hyper-responsiveness. Although most asthma patients can achieve symptom control with regular controller treatment, $5-10 \%$ of them suffer from a severe form of asthma that is uncontrolled despite maximal pharmacological therapy. Severe asthma patients have asthma with exacerbations even if under treatment with high dose inhaled corticosteroids (ICS) together with beta-2 long-acting bronchodilators (LABA) and ipratropium bromide as a long-acting muscarinic antagonist. They also often need oral corticosteroids (OCS) as an add on treatment in order to control asthma symptoms. ${ }^{1}$

Severe Allergic Asthma (SAA) is a severe asthma phenotype with a clinically relevant allergic sensitization, ${ }^{2}$ which can be treated with omalizumab, an anti-IgE monoclonal antibody, as an add-on treatment which has shown to reduce the rate of asthma exacerbations. ${ }^{3}$ 
Different psychological factors have been reported to play a primary role in daily asthma management; amongst them alexithymia has been reported to influence the achievement of disease control. ${ }^{4}$

Asthmatic patients with alexithymia tend to perceive and to live their disease as a cyclical disorder, not a chronic condition; they have difficulty in recognizing and in communicating body sensations and emotional perceptions and tend to underestimate both physical and emotional components of asthma exacerbations. ${ }^{5}$ As a result, it has been reported that asthma patients with alexithymia have a suboptimal control of asthma, an increased risk of asthma exacerbations and they could suffer more severe asthma attacks, including near fatal asthma. ${ }^{6}$

A study was conducted to evaluate the presence of alexithymia in patients with SAA treated with omalizumab in order to determine if this condition influences the clinical efficacy of omalizumab treatment and the patients' quality of life (QoL).

\section{Methods}

Data were collected from 18 consecutive patients who were diagnosed SAA after referring to the Asthma Center of the Allergy Unit in Messina University Hospital "G.Martino" over a two-year-treatment period with omalizumab.

Patients' characteristics at the beginning of the study were:

- severe persistent allergic asthma for over 12 months, not controlled by maximum asthma drug treatment;

- documented repeated severe asthma exacerbations and need for OCS;

- evidence of positivity at skin prick tests and/or laboratory tests for at least one perennial allergen, such as dust mites and/or parietaria pollen, perennial in the Mediterranean area; ${ }^{7}$

- total serum IgE levels $\geq 30 \mathrm{UI} / \mathrm{ml}$ and $\leq 1500 \mathrm{UI} / \mathrm{ml}$;

- basal FEV $1<80 \%$

Omalizumab treatment was prescribed according to patients' total IgE levels and body weight every 2 or 4 weeks with doses ranging from 150 to $1200 \mathrm{mg}$ monthly.

Patients were evaluated before starting treatment with omalizumab (T0) and after 1 (T1) and 2 years (T2).

Number of asthma exacerbations were recorded and pulmonary function was evaluated with spirometry measuring the forced expiratory volume in 1 second \% (FEV1\%).

Patients' asthma symptom control was assessed using the asthma control test (ACT), a 5-item questionnaire about asthma impact on home and work activities, shortness of breath, symptoms, rescue medication usage, and overall asthma control. Scores range from 5-25 (higher is better). Scores of 20-25 are classified as well-controlled asthma; $16-19$ as not well-controlled; and $5-15$ as very poorly controlled asthma. ${ }^{8}$
Patients' quality of life (QoL) was measured by the standardized version of the asthma quality of life questionnaire (AQLQ) which consists of 4 domains (symptoms, activity limitation, emotional function and environmental stimuli), with a total of 32 items; the overall score is the mean of these 32 items on a scale of 1 to 7 $(1$ = severe impairment; 7 = no impairment $) .{ }^{9}$

The presence of alexithymia was measured by the total alexithymia score (TAS), a self-report scale that is comprised of 20 items, rated using a 5-point Likert scale whereby $1=$ strongly disagree and $5=$ strongly agree. There are 5 items that are negatively keyed (items 4, 5, 10, 18 and 19).

At T0 Group 1 and Group 2 parameters had no statistical difference as reported in Table $\mathbf{1}$.

Table 1. Comparison of patient characteristics between the two groups

\begin{tabular}{|c|c|c|c|}
\hline $\begin{array}{c}\text { At T0 } \\
\text { (baseline) }\end{array}$ & $\begin{array}{c}\text { TAS } 20 \\
\text { \{greater than } \\
\text { or equal to\} } 61\end{array}$ & $\begin{array}{c}\text { TAS } 20 \\
\text { \{less than or } \\
\text { equal to\} } 51\end{array}$ & $p$ \\
\hline Age* & $44.5 / 8.75$ & $42 / 18.5$ & n.s. \\
\hline Male/Female & $4 / 2$ & $4 / 8$ & n.s. \\
\hline Asthma exacerbations ${ }^{*}$ & $13.5 / 14$ & $20 / 12$ & n.s. \\
\hline $\mathrm{ACT}^{*}$ & $12 / 6.5$ & $11 / 7.5$ & n.s. \\
\hline FEV $1 \% *$ & $69.35 / 18.5$ & $61.85 / 13.75$ & n.s. \\
\hline OCS & $6 / 6$ & $12 / 12$ & n.s. \\
\hline ICS & $6 / 6$ & $12 / 12$ & n.s. \\
\hline LABA & $6 / 6$ & $12 / 12$ & n.s. \\
\hline Antileukotrienes & $5 / 6$ & $8 / 12$ & n.s. \\
\hline Levels of IgE UI/ml ${ }^{*}$ & $408.5 / 479$ & $467 / 553$ & n.s. \\
\hline Eosinophils/ $\mu \mathrm{L}^{*}$ & $150 / 295$ & $295 / 310$ & n.s. \\
\hline \multicolumn{4}{|l|}{ At T1 (1 year) } \\
\hline Asthma exacerbations ${ }^{\star}$ & $3.5 / 12.5$ & $3.5 / 5$ & n.s. \\
\hline $\mathrm{ACT}^{*}$ & $20 / 5.5$ & $18.5 / 5.75$ & n.s. \\
\hline FEV1\%* & $78 / 19.85$ & $73.5 / 23$ & n.s. \\
\hline OCS & $2 / 6$ & $3 / 12$ & n.s. \\
\hline ICS & $6 / 6$ & $12 / 12$ & n.s. \\
\hline LABA & $6 / 6$ & $12 / 12$ & n.s. \\
\hline Antileukotrienes & $2 / 6$ & $3 / 12$ & n.s. \\
\hline Levels of IgE UI/ml* & $325 / 454$ & $375 / 421$ & n.s. \\
\hline Eosinophils $/ \mu L^{*}$ & $180 / 100$ & $245 / 183$ & n.s. \\
\hline
\end{tabular}


Table 1. (Continued)

\begin{tabular}{|l|c|c|c|}
\multicolumn{1}{c|}{$\begin{array}{c}\text { At T0 } \\
\text { (baseline) }\end{array}$} & $\begin{array}{c}\text { TAS 20 } \\
\text { \{greater than } \\
\text { or equal to } \text { 61 }\end{array}$ & $\begin{array}{c}\text { TAS 20 } \\
\text { \{less than or } \\
\text { equal to } 51\end{array}$ & $p$ \\
\hline At T2 (2 years) & $2.5 / 8.75$ & $0 / 1.75$ & n.s. \\
\hline Asthma exacerbations* & $20.5 / 8.5$ & $21.5 / 2.5$ & n.s. \\
ACT $^{*}$ & $81.5 / 23.75$ & $80.5 / 26.75$ & n.s. \\
FEV1\%* $^{*}$ & $2 / 6$ & $3 / 12$ & n.s. \\
OCS & $6 / 6$ & $12 / 12$ & n.s. \\
ICS $^{*}$ & $6 / 6$ & $12 / 12$ & n.s. \\
\hline LABA & $4 / 6$ & $3 / 12$ & n.s. \\
\hline Antileukotrienes & $281 / 455$ & $324 / 223$ & n.s. \\
\hline Levels of IgE UI/ml* & $195 / 230$ & $175 / 203$ & n.s. \\
\hline Eosinophils/ $\mu L^{*}$ &
\end{tabular}

* Data are presented as median / interquartile range (IQR).

Abbreviations: ACT: asthma control test; FEV1\%: forced expiratory volume in 1 second \%; OCS: oral corticosteroids; ICS: inhaled corticosteroids; LABA: long-acting beta2 agonist

All subjects provided written informed consent for processing of personal data according to GDPR 2016/679.

Data reported in this study are from the SANI (Severe Asthma Network in Italy) section of G.Martino University Hospital of Messina approved from the Messina Ethical Committee on the 18 March of 2019, protocol n. 0002709.

\section{Statistical analysis}

Statistical analysis was performed with the SPSS program. Data are presented as median and interquartile range (IQR).

Comparisons between categorical variables (sex differences in the two groups) were performed with the exact Fisher test.
Comparisons between the two groups for T0 variables of asthma exacerbations, ACT, FEV1\%, AQLQ related to symptoms, activity limitations, emotional functions, environmental stimuli and TAS were calculated using the Mann-Whitney Test.

Comparisons between variables taken into consideration in the two groups (exacerbations, ACT, FEV1\%, AQLQ) at T0, $\mathrm{T} 1$ and $\mathrm{T} 2$ were calculated with the Friedman test; between $\mathrm{T} 0$ and $\mathrm{T} 1$ and finally between $\mathrm{T} 1$ and $\mathrm{T} 2$ were calculated with the Wilcoxon Signed Ranks Test.

Statistical significance was set for $p<0.05$.

\section{Results}

According to the TAS results patients were divided into two groups: group 1 (TAS $20 \geq 61$ ) resulted of 6 patients with alexithymia (2 males and 4 females; median age 44.5 years), group 2 (TAS $20 \leq 51$ ) resulted of 12 patients without alexithymia ( 8 males and 4 females; median age 42 years). The TAS 20 score is the sum of responses to all 20 items. It uses cutoff scoring: equal to or less than $51=$ non-alexithymia, equal to or greater than $61=$ alexithymia, scores of 52 to $60=$ possible alexithymia. ${ }^{10}$

In our study no Group 1 patient had TAS scores between 50 and 60 . Patients with alexithymia were categorized according to Factor 1 to 3 TAS questions, as already done by Bagby et al. ${ }^{10}$ differences on difficulty identifying feeling subscale Factor 1 were significant but not for Factor 3; Factor 2, among difficulty describing feeling subscale, was also significant.

At T0 no differences in values of asthma exacerbations (Figure 1), ACT (Figure 2), FEV1\% (Figure 3), AQLQ scores (Figure 4) related to symptoms, emotional function and environmental stimuli were found; instead, significantly lower AQLQ activity limitation scores were recorded in group 1 with alexithymia compared to group 2 (Figure 4).

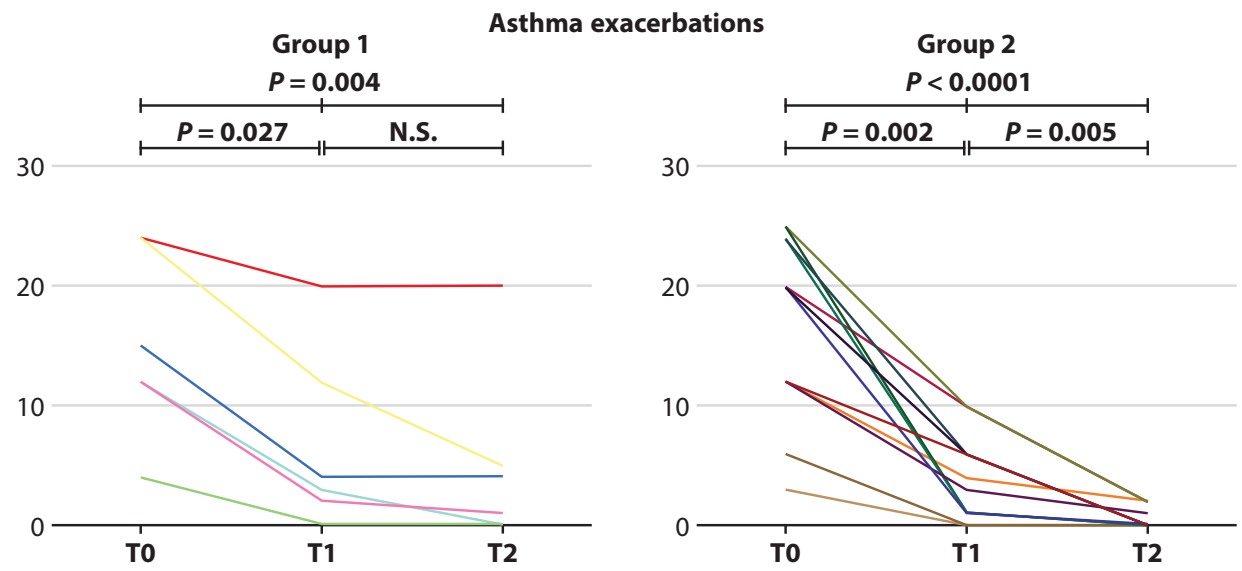

Figure 1. Asthma exacerbations in group 1 and group 2 patients with or without alexithymia respectively. 

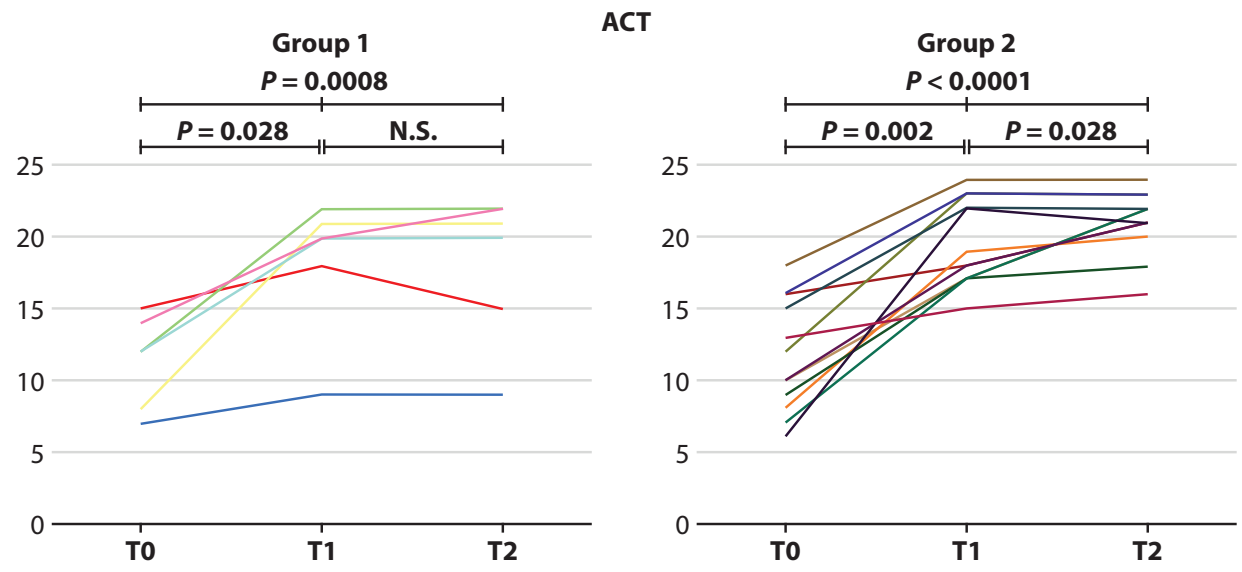

Figure 2. Asthma Control Test (ACT) scores in group 1 and group 2 patients with and without alexithymia respectively.

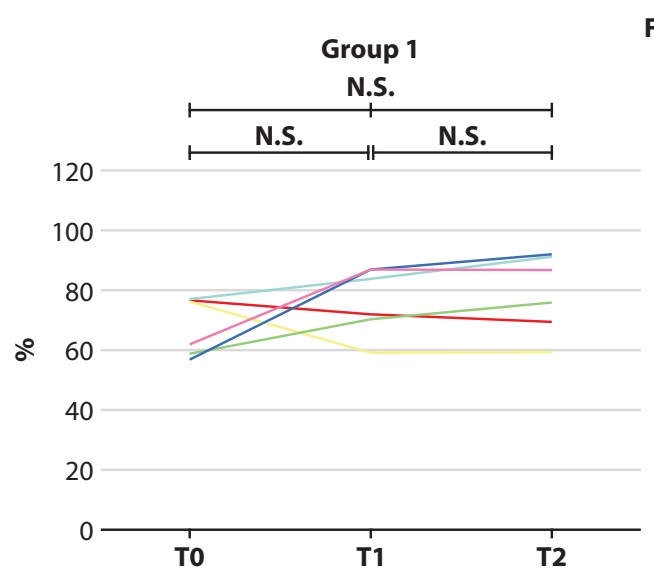

FEV1

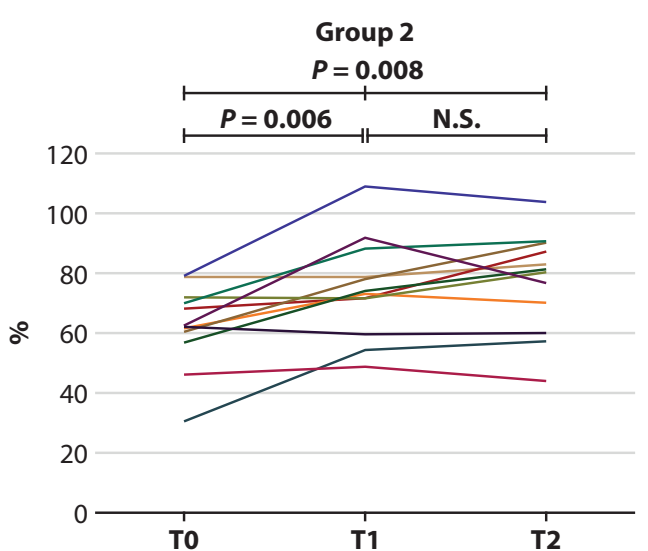

Figure 3. FEV1\% values in group 1 and group 2 patients with and without alexithymia respectively.

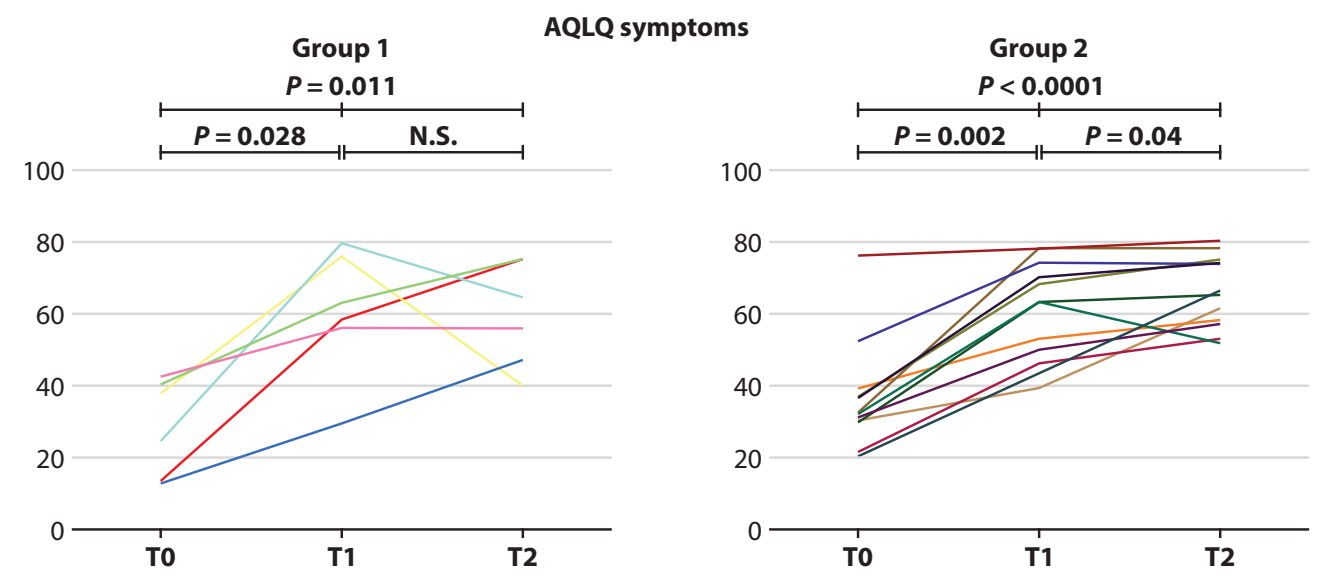

Figure 4. Asthma quality of life questionnaire (AQLQ) scores for symptoms, activity limitation, emotional function, environmental stimuli in group 1 and group 2 patients with and without alexithymia respectively. 


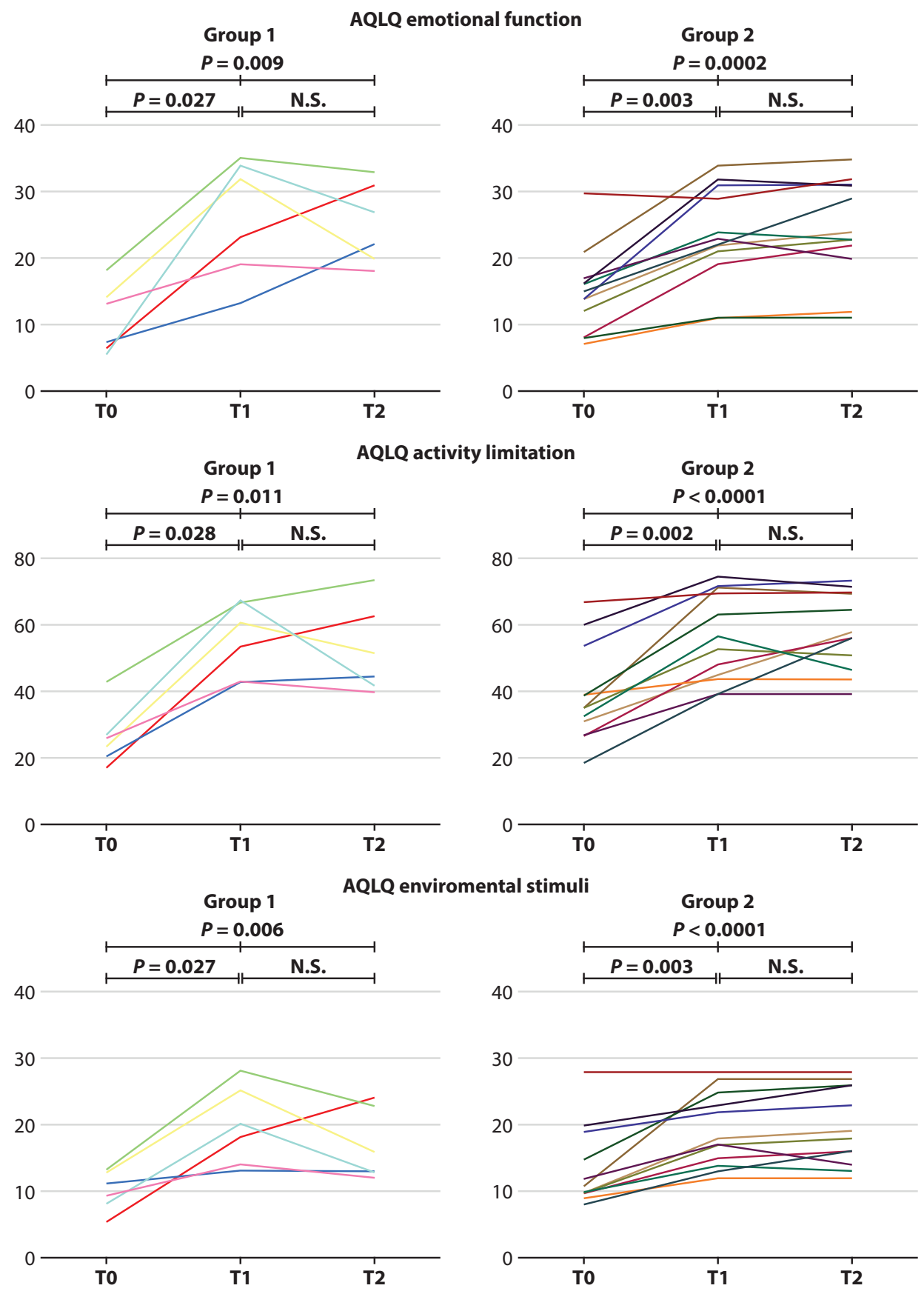

Figure 4. (Continued)

The effects of the drug at $\mathrm{T} 1$ and $\mathrm{T} 2$ were evaluated in Group 1 and 2 subjects. The results are reported in color graphs where each color indicates the same patient in the different graphs (Figure 1, 2, 3, 4).

Group 1 patients had a statistically significant decrease between $\mathrm{T} 0$ and $\mathrm{T} 2$ in the number of asthma exacerbations (Figure 1) while ACT (Figure 2) and AQLQ scores related to symptoms, activity limitation, emotional function and environmental stimuli increased significantly (Figure 4). FEV1 values (Figure 3) from T0 to T2 also increased in group 1 but not statistically significantly.
As the level of $\operatorname{IgE}$ is very important for the effects of omalizumab the analysis between IgE and effects of omalizumb was performed; Group 1 patients had a decrease between T0 and T2 in the level of IgE, although not statistically significant, while in group 2 the decrease was statistically significant $(p=0.028)$.

Both groups showed a marked improvement in all the parameters and there were no substantial differences in the trend of the single variables in the two groups for the three intervals of time considered and therefore no statistically significant differences in treatment outcomes were recorded between group 1 and group 2 patients (Table 1). 


\section{Discussion}

Alexithymia has been reported as a possible risk factor for a variety of chronic diseases. ${ }^{11}$ The term alexithymia was coined by Sifneos from the Greek 'a' for lack, 'lexis' for word and 'thymos' for emotion with the meaning of lack of words for emotions and it refers to a personality construct characterized by difficulty in identifying and describing feelings and distinguishing them from bodily sensations. ${ }^{12}$

The prevalence of alexithymia in asthmatic patients seems to be higher compared to the general population as asthma, an inflammatory chronic disease of the lower airway, has been reported to be associated to alexithymia in almost 1 patient out of 5 in real life studies. ${ }^{13}$

Our study on patients with severe allergic asthma confirms these data as alexithymia resulted in one third of our patients that is in 6 out of 18 .

Asthma patients have been reported to underestimate the severity of their disease due to the inability in recognizing and distinguishing symptoms from emotional perceptions. ${ }^{14}$ This condition in patients with asthma and alexithymia may even lead to near-fatal asthma an acute respiratory insufficiency attack occurring as a consequence of chronic hyperreactivity typical of asthma. ${ }^{15}$

It is likely that in our study asthma patients with alexithymia (Group 1) had a worse QoL, highlighted by lower activity limitation AQLQ scores at T0, as they did not accept to limit their activities because of an incorrect perception of their respiratory symptoms, risking more easily a severe asthma attack. ${ }^{16}$

Regardless the presence or not of alexithymia, all the patients included in our study obtained a marked improvement of all the parameters after starting treatment with omalizumab, which is an established SAA treatment. ${ }^{17}$ In particular, both groups showed a statistically significant improvement of exacerbations, ACT and AQLQ scores since the first year of treatment which was maintained constantly over time.

As regards FEV1, we found a statistically significant increase in the group with no alexithymia and a marked, but not statistically significant, increase in patients with alexithymia; it was ruled out that patients with alexithymia, while continuing omalizumab treatment, discontinued standard asthma treatment with ICS + Beta2 long-acting agents because of their psychological status. It is possible to hypothesize that SAA patients with alexyithymia had previously neglected their disease causing repeated airway epithelium injury because of chronic inflammation and had become more conscious of their disease only after the improvement of asthma symptoms and QoL induced by omalizumab. ${ }^{18}$

Probably the inclusion in our study of patients with asthma and alexithymia following a treatment plan with scheduled visits as well as an add-on treatment with omalizumab, a biological drug with diseases modifying properties, encouraged the establishment of a relationship of trust between physicians and patients. ${ }^{19}$ This increased awareness in patients of their illness and counteracted the tendency of asthma patients with alexithymia to poor treatment adherence. ${ }^{20}$
Therefore, it could be useful to assess the presence of alexithymia in patients with asthma especially in patients with severe asthma, allergic or not, in order to plan an adequate disease management at regular and close time intervals.

The results of our study confirm the efficacy of omalizumab in the treatment of SAA, in terms of greater control of symptoms, improvement of respiratory function and QoL introducing an original aspect as it shows the efficacy of omalizumab regardless the presence or not of alexythimia. Nevertheless, it must be underlined that further studies on wider populations could implement these data.

Studies carried out on patients with alexithymia suffering from other chronic diseases ${ }^{21}$ report that the improvement of alexithymia after psychotherapeutic interventions seems to influence the clinical course of the underlying disease. It would therefore be interesting to evaluate whether the introduction of psychotherapeutic treatment in the management of asthma patients with alexithymia could further improve the pharmachological induced control of asthma.

\section{Aknowledgements}

All the authors have no conflict of interest to declare.

\section{Authors' contribution}

- ML, ML and LR followed thr patients, designed and described the study.

- SS performed the statistical analysis.

- All authors approved the final manuscript.

\section{References}

1. Global initiative for Asthma [Internet]. Fontana: Global Initiative for Asthma; c2018.2018 GINA Report, Global Strategy for Asthma Management and Prevention [cited 2018 Apr 28]; [about 1 screen]. Available from: http://ginasthma.org/2018-ginareport-global-strategy -forasthma-management-and-prevention/.

2. Holt PG, Macaubas C, Stumbles PA, Sly PD. The role of allergy in the development of asthma. Nature. 1999;402:12-7.

3. Agache I, Beltran J, Akdis C, Akdis M, Canelo-Aybar C, Canonica GW, et al. Efficacy and safety of treatment with biologicals (benralizumab, dupilumab, mepolizumab, omalizumab and reslizumab) for severe eosinophilic asthma. A systematic review for the EAACI Giudelines-recommendations on the use of biologicals in severe asthma. Allergy. 2020;75:1023-42.

4. Baiardini I, Sicuro F, Balbi F, Canonica GW, Braido F. Psychological aspects in asthma: do psychological factors affect asthma management? Asthma Res Pract. 2015;1:7.

5. Amore M, Antonucci C, Bettini E, Boracchia L, Innamorati M, Montali A, et al. Disease control in patients with asthma is associated with alexithymia but not with depression or anxiety. Behav Med. 2013;39: 138-45.

6. Chugg K, Barton C, Antic R, Crockett A. The impact of alexithymia on asthma patient management and communication with health care providers: a pilot study. J Asthma. 2009;4682:126-9.

7. Sala-Cunill A, Bartra J, Dalmau G, Tella R, Botev E, Raga E, et al. Prevalence of Asthma and Severity of Allergic Rhinitis Comparing 2 Perennial Allergens: House Dust Mites and Parietaria Judaica Pollen. J Invest Allergol Clin Immunol. 2013;23:145-51.

8. Nathan RA, Sorkness CA, Kosinski M, Schatz M, Li JT, Marcus P, et al Development of the asthma control test: a survey for assessing asthma control. J Allergy Clin Immunol. 2004;113:59-65. 
9. Juniper EF, Buist AS, Cox FM, Ferrie PJ, King DR. Validation of a standardized version of the Asthma Quality of Life Questionnaire. Chest. 1999;115:1265-70.

10. Bagby RM, Parker JD, Taylor GJ. The twenty-item Toronto Alexithymia Scale Item selection and cross-validation of the factor structure. J Psychosom Res. 1994;38:23-32.

11. Wrienga KL, Lehto RH, Given B. Emotion regulation in Chronic disease populations: an integrative review. Theory Nurs Pract. 2017;31:247-71.

12. Chung MC, Rudd H, Wall N. Posttraumatic stress disorder following asthma attack (post-asthma attack PTSD) and psychiatric co-morbidity: the impact of alexithymia and coping. Psychiatry Res. 2012;197(3): 246-52.

13. Baiardini I, Braido F, Ferraioli G, Menoni S, Bruzzone M, Conte ME, et al. Pitfalls in respiratory allergy management: alexithymia and its impact on patient reported outcomes. J Asthma. 2011;48:25-32.

14. Bidder TM. Effective management of adult patients with asthma. Nurs Stand. 2019;34:43-50.
15. Restrepo RD, Peters J. Near-fatal asthma:recognition and management. Curr Opin Pulm Med. 2008;14:13-23.

16. Serrano J, Plaza V, Sureda B, de Pablo J, Picado C, Bardagì S, Lamela J, et al. Alexithymia: a relevant psychological variable in near-fatal asthma. Eur Respir J. 2006;28:296-302.

17. Katsaounou P, Buhl R, Brusselle G, Pfister P, Martinez R, Wahn U, et al. J Respir Med 2019;150:51-62

18. Shinan-Altman S, Katzav KO. The relationship between illness representations, alexithymia, coping strategies and subjective well-being among persons with asthma. J Asthma. 2020;16:1-7.

19. Domingo C. Omalizumab for severe asthma: efficacy beyond the atopic patient? Drugs. 2014;74:521-33

20. Axelsson M, Emilsson M, Brink E, Lundgren, Toren $\mathrm{K}$, Lötvall J. Personality, adherence, asthma control and health-related quality of life in young adult asthmatics. Respir Med. 2009;103:1033-40.

21. Beresnevaite M. Exploring the benefits of group psychotherapy in reducing alexithymia in coronary heart disease patients: A preliminary study. Psychother Psychosom. 2000;69:117-22. 